\title{
Digitalisierung von illegalen Märkten
}

\author{
Folgen, Grenzen und Perspektiven
}

\author{
Meropi Tzanetakis
}

\section{Zusammenfassung}

Der Beitrag untersucht sozioökonomische Veränderungen von Drogenmärkten durch die Verbreitung digitaler Technologien. Dabei steht vor allem das neuartige Phänomen der Kryptomärkte im Fokus. Durch die Verwendung von Anonymisierungssoftware können Handelnde auf Plattformen im Internet ihre Sichtbarkeit erhöhen, ohne dass gleichzeitig das Risiko der Strafverfolgung steigt. Hinzu kommt, dass auf Kryptomärkten anstelle des interpersonellen ein institutionenbasiertes Vertrauen hergestellt wird, etwa durch Feedback- oder Bezahlsysteme. Wie in modernen kapitalistischen Ökonomien tragen diese Entwicklungen sowie die orts- und zeitunabhängige Verfügbarkeit von illegalen Waren zu einer Ausweitung von Marktbeziehungen bei und fordern gleichzeitig globale Prohibitionsregime heraus.

Schlüsselbegriffe: Drogenhandel, Kryptomärkte, digitale Technologie, Drogenmärkte, Internet

Anders als die weitläufige mediale Berichterstattung zum Thema Drogenvertrieb und Internet zunächst vermuten lässt, stellt der Verkauf und Kauf von legalen und illegalen Drogen im Internet kein neues Phänomen dar, sondern findet sich bereits in den Anfängen des Netzes (vgl. Buxton und Bingham 2015; Martin 2014). Als jedoch im Februar 2011 die digitale Plattform Silk Road online ging, erreichte der Handel von psychoaktiven Substanzen eine neue Dimension. Die grenzenlose Verfügbarkeit sämtlicher psychoaktiver Substanzen, sowie der niederschwellige Zugang und die Anonymität der beteiligten Akteur_innen waren einige der wichtigsten Neuerungen. Der Aufbau und die anwenderfreundlichen Bedienungselemente von Silk Road erinnerten an bekannte E-Commerce-Plattformen wie Amazon Marketplace oder Ebay. Erstmals konnte im Gegensatz zum Drogenvertrieb im

Die Originalversion dieses Kapitels wurde revidiert. Ein Erratum ist verfügbar unter https://doi.org/10.1007/978-3-658-22138-6_47

(C) Der/die Autor(en) 2019 
Clearnet (dem über gängige Suchmaschinen erfassbaren Internet) auf dieser neuen Plattform der gesamte Verkauf abgewickelt werden. Das schloss sowohl die Kommunikation zwischen Drogenhändler_in und Kund_innen als auch die Zahlungsabwicklung ein. Allerdings war das Angebot auf Silk Road nicht auf psychoaktive Substanzen beschränkt und umfasste ein breites Spektrum an Waren und Dienstleistungen wie verschreibungspflichtige Arzneimittel, pornografisches Material, gefälschte Dokumente (etwa Führerscheine oder Personalausweise), Hacking-Anleitungen, Turnschuhe oder Sonnenbrillen.

Grundsätzlich kann der bzw. die Betreiber_in einer Plattform festlegen, welche Produkte und Services verkauft werden dürfen. Der_die Administrator_in von Silk Road hat entsprechende Einschränkungen in seinen Geschäftsbedingungen festgelegt: „Do not list anything who's (sic) purpose is to harm or defraud, such as stolen items or info, stolen credit cards, counterfeit currency, personal info, assassinations, and weapons of any kind. Do not list anything related to pedophilia“" (Silk Road, zitiert nach Christin 2013, S. 3). Aus dem Beispiel wird ersichtlich, dass auf Silk Road kein kinderpornographisches Material vertrieben werden durfte - eine Norm, die sich seither weitgehend in der Kryptomarkt-Community gehalten hat (vgl. Martin 2014). Als Silk Road im Oktober 2013 vom FBI geschlossen wurde, eröffneten zahlreiche neue Plattformen, wovon einige im Laufe der Zeit von Strafermittlungsbehörden oder seitens der bzw. die Betreiber_in wieder geschlossen wurden (vgl. Tzanetakis und Stöver 2018). Aktuell sind etwa ein Dutzend Kryptomärkte online, die sich in Bezug auf Größe, Sprache, Bezahlsystem, Lebensdauer sowie Ausschluss oder Ermöglichung des Vertriebs von Waffen unterscheiden (vgl. Darknet Stats 2018).

Der vorliegende Beitrag ${ }^{1}$ beschäftigt sich mit der Frage, welche sozialen und ökonomischen Folgen, Grenzen und Perspektiven die Verbreitung digitaler Technologien für illegale Drogenmärkte haben. Um diese Frage systematisch zu beantworten, wird zunächst der Begriff Kryptomärkte geklärt und anschließend ein Überblick über den sozialwissenschaftlichen Forschungsstand zu diesem neuartigen Phänomen gegeben. Im nächsten Schritt werden fünf grundlegende Veränderungsprozesse durch die Digitalisierung von Märkten für illegale Waren herausgearbeitet. Außerdem wird beleuchtet, welche Effekte diese auf materiellen Drogenmärkten nach sich ziehen. Abschließend wird ein Ausblick auf weitere Entwicklungsperspektiven gegeben, und es werden mögliche Regulierungsansätze diskutiert.

\section{$1 \quad$ Kryptomärkte. Eine Definition}

Als Kryptomärkte werden Plattformen im Internet bezeichnet, die zwei wesentliche Elemente miteinander kombinieren: Anonymisierungssoftware und virtuelle Währungen (vgl. Barratt et al. 2014; Martin 2014). Erstens verwenden Kryptomärkte eine Verschlüs-

1 Fußnote: Gefördert durch den Austrian Science Fund (FWF): Projektnummer J4095-G27. 
selungssoftware (z.B. den TOR ${ }^{2}$-Browser), mit der sogenannte hidden services (versteckte Dienste) aufgerufen werden können und damit der Zugang zum Darknet (ein kleiner Teil des Internets, in dem Nutzer_innen nahezu komplett anonym kommunizieren können) ermöglicht wird. Durch die Anonymisierungssoftware werden der Datenverkehr in der Kommunikation zwischen den beteiligten Akteur_innen verschlüsselt und Identität sowie Standort der Benutzer_innen mittels technischer Lösungen verschleiert.

Die zweite Innovation von Kryptomärkten liegt in der Nutzung virtueller Währungen zur Zahlungsabwicklung (in erster Linie Bitcoin oder Monero). Während zuvor Drogen-Bestellungen über das Internet mit Western Union, PayPal oder Bargeld in Briefsendungen bezahlt wurden (vgl. Buxton und Bingham 2015), werden auf anonymen Plattformen im Darknet sogenannte Kryptowährungen wie Bitcoin genutzt, die den Nutzer_innen pseudonyme und dezentrale Transaktionen ermöglichen (vgl. Tzanetakis 2016b). Dezentral bedeutet, dass diese virtuellen Zahlungsmittel nicht von staatlichen Institutionen ausgegeben werden. Virtuelle Währungen erschweren die Rückverfolgbarkeit, weil keine unmittelbare Verbindung zwischen der Identität der Transaktionspartner_innen und den Bitcoins besteht. Allerdings wird bei Bitcoin die Historie sämtlicher Zahlungsabwicklungen in der sogenannten Blockchain gespeichert, die eventuell für strafrechtliche Ermittlungen verwendet werden kann. Entscheidend ist aber, wie konsequent die Verbindung zwischen der Identität und den Bitcoins verschleiert werden kann, etwa beim Tausch von Euro in Bitcoin. Die Kombination dieser beiden technologischen Innovationen ermöglichte einen systematischen Drogenhandel im Internet.

Weil der Begriff Darknet im öffentlichen Diskurs mit Kriminalität verknüpft ist, hat sich in der Forschungsgemeinschaft der neutralere Begriff Kryptomärkte durchgesetzt. Darknet bezeichnet lediglich die Art und Weise des Zugangs (über eine Anonymisierungssoftware) und sagt nichts über den rechtlichen Status der Inhalte aus (vgl. Tzanetakis 2018a). Im vorliegenden Kapitel werden die Begriffe Kryptomärkte, Darknet-Drogenmärkte und anonyme Drogenmarktplätze deshalb synonym verwendet.

\section{Sozialwissenschaftlicher Forschungsstand zu Kryptomärkten}

Seit dem erstmaligen Auftreten von Kryptomärkten im Jahre 2011 haben Sozialwissenschafler_innen zu dem neuartigen Phänomen geforscht. Bisherige Studien zum Forschungsfeld lassen sich in drei Stränge einteilen: a) Forschungsarbeiten zur allgemeinen Struktur der anonymen Drogenmarktplätze, b) Arbeiten zur Prävalenz des Drogenkonsums und zur Zahl der Nutzer_innen der Marktplätze und c) Forschungsarbeiten, die sich mit der Rolle einzelner Industriestaaten auf Kryptomärkten befassen und Implikationen für Harm Reduction-Ansätze (Schadensminimierung) diskutieren.

2 Anm.: TOR steht für The Onion Router. TOR basiert auf einem Netzwerk an weltweit rund 7000 Servern, wobei Anfragen verschlüsselt über drei zufällig gewählte Server geleitet werden und damit die Kommunikation innerhalb des Netzwerks kaum zurückverfolgt werden kann. 
(a) Quantitative Erhebungen haben dazu beigetragen, die allgemeine Bedeutung und Struktur von Kryptomärkten einzuschätzen. Messungen materieller Drogenmärkte (physischer Austausch der Waren) bleiben aufgrund des Dunkelfeldcharakters ihres Forschungsfeldes auf Beschlagnahmungen, Satellitenaufnahmen und Zahlen der Substitutionspatienten beschränkt. Auch auf Online-Märkten besteht eine schwer schätzbare Dunkelziffer, allerdings können halb-öffentlich verfügbare Marktdaten auf anonymen Drogenmarktplätzen systematischer extrahiert werden. Damit lässt sich das Handelsgeschehen von Kryptomärkten nicht vollständig, aber zumindest verlässlicher abbilden (vgl. Tzanetakis 2018b). Umsätze können beispielsweise errechnet werden, indem das Feedback der User_innen mit dem Preis eines Drogenangebots multipliziert wird. Big Data, Web Scraping und Web Crawling sind die Methoden, mit denen in diesem Bereich Daten gesammelt werden.

In der ersten quantitativen Erhebung hat Christin (2013) das Umsatzvolumen im Jahre 2012 für den damaligen Monopolisten Silk Road auf rund 15 Millionen US-Dollar geschätzt. Der Umsatz ist laut einer weiteren Studie im Jahr 2013 auf mehr als 100 Millionen US-Dollar angestiegen (vgl. Soska und Christin 2015). Aldridge and Décary-Hétu (2014) kamen in ihrer quantitativen Erhebung zu dem Schluss, dass die Anzahl der Verkäufer_innen, die Zahl der Transaktionen und folglich auch die Umsätze im September 2013 - kurz vor der Schließung von Silk Road - nochmals angestiegen sind. Dennoch ist das Umsatzniveau von 2013, so das Ergebnis einer Langzeitstudie, auch zwischen 2015 und 2016 konstant geblieben (vgl. Tzanetakis 2018b). Der ehemalige Marktführer AlphaBay hat demnach zwischen September 2015 und August 2016 einen Umsatz von rund 94 Millionen US-Dollar erzielt, wobei diese Schätzung im Gegensatz zu anderen Erhebungen lediglich Drogenangebote umfasst. Die Zahlen verdeutlichen, dass es zu Beginn einen deutlichen Umsatzzuwachs auf den Kryptomärkten gab, die Umsätze seit 2013 allerdings ein relativ stabiles Niveau erreicht haben. Ebenso zeigt ein Vergleich mit dem materiellen Einzelhandelsmarkt für illegale Drogen in Europa, der auf rund 28 Milliarden US-Dollar geschätzt wird (vgl. EMCDDA 2016), dass Kryptomärkte momentan nur einen Bruchteil hiervon ausmachen.

(b) Ein zweiter Forschungsstrang beschäftigt sich mit den Nutzer_innen von Kryptomärkten und der Prävalenz des Drogenkonsums. Bisherige Forschungen konnten unabhängig vom Kryptomarkt und Forschungsdesign zeigen, dass Kund_innen und Händler_ innen überwiegend männlich und zwischen 20 bis 40 Jahre alt sind, einer Erwerbstätigkeit nachgehen oder eine Hochschulausbildung absolvieren und technologisch gebildet sind (vgl. Bancroft und Reid 2016; Barratt et al. 2016a, 2016b; Buxton und Bingham 2015; Kruithof et al. 2016; Ormsby 2016; van Buskirk et al. 2016; van Hout und Bingham 2013a, 2013b). Die populärsten auf Kryptomärkten gehandelten Drogen sind Cannabis, Ecstasy und Kokain bzw. verwandte Substanzen (vgl. Aldridge und Décary-Hétu 2016; Barratt et al. 2014; 2016b; Demant et al. 2016; Soska und Christin 2015; Tzanetakis 2018b; van Buskirk et al. 2016; van Hout und Bingham 2013a, 2013b).

Die Mehrzahl der Kund_innen auf Kryptomärkten konsumieren die Substanzen in ihrer Freizeit, vorwiegend am Wochenende (vgl. Barratt et al. 2014; Ormsby 2016; van 
Hout und Bingham 2013b). Der Global Drug Survey zufolge konsumiert 59,3\% der Nutz_ innen zumindest einmal monatlich auf Partys, wobei davon $21 \%$ zumindest einmal in der Woche psychoaktive Substanzen konsumieren (vgl. Barratt et al. 2016b). Damit verfügt das Hauptzielpublikum von anonymen Drogenmärkten im Internet über die nötigen finanziellen und technischen Ressourcen, hat die entsprechenden Kompetenzen und die Zeit, um online Drogen zu bestellen und auf die Lieferung per Postdienst zu warten. Impulsgesteuerte Konsumierende verfügen in der Regel über keine dieser Ressourcen und Fähigkeiten.

Die Mehrzahl der Verkäufer_innen (etwa 70\%) wiederum konnte laut zweier Langzeitstudien Waren im Wert von weniger als 1000 US-Dollar verkaufen (vgl. Soska und Christin 2015; Tzanetakis 2018b). Nur etwa 5 Prozent der Händler_innen machen einen Umsatz von mehr als 200.000 US-Dollar. Ein Großteil der Händler_innen auf Kryptomärkten vertreibt also Drogen im kleinen Stil, während lediglich ein kleiner Anteil hohe Umsätze erzielt.

Aldridge und Décary-Hétu $(2014,2016)$ waren die ersten, die sich mit der Frage beschäftigten, in welchem Ausmaß die auf Kryptomärkten erworbenen Drogen auf materiellen Märkten wiederzufinden sind. Etwa ein Viertel der auf dem Marktplatz Silk Road erzielten Umsätze wird mit Transaktionen gemacht, die vermutlich zur Weitergabe bestimmt sind (vgl. Aldridge und Décary-Hétu 2016; Barratt et al. 2016b; Demant et al. 2016; Tzanetakis 2018b). Allerdings bleibt unklar, in welcher Form die Substanzen weitergegeben werden: $\mathrm{Ob}$ es sich um einen profitorientierten Weiterverkauf handelt, die Finanzierung des eigenen Konsums oder um eine unentgeltliche Weitergabe an Freunde oder Bekannte, bedarf weiterer Forschung (vgl. Coomber et al. 2016; Reuter 1983; Sandberg 2012; Taylor und Potter 2013; Werse und Bernard 2016).

(c) Ein dritter Forschungsstrang beschäftigt sich mit der Bedeutung von Kryptomärkten für die jeweiligen Industrienationen und der Relevanz des neuartigen Phänomens für Harm Reduction. Auf Kryptomärkten machen die Verkäufer_innen neben Angaben zur Substanz, Qualität, Menge und Preis für gewöhnlich auch Angaben zum Herkunftsland. Diese Information dient den Kund_innen zur Risikoeinschätzung bei der Lieferung. So ist z.B. die Wahrscheinlichkeit höher, dass eine Drogenlieferung per Postsendung aus England ankommt als eine aus Kolumbien. Diese Angaben konnten für Forschungszwecke verwendet und systematisch ausgewertet werden. Die fünf häufigsten Herkunftsländer sind die Vereinigten Staaten, gefolgt von Großbritannien, Australien, den Niederlanden und Deutschland (vgl. Aldridge und Décary-Hétu 2016; Christin 2013; Kruithof et al. 2016; Tzanetakis 2018b; van Buskirk et al. 2016). Damit zeigt sich die Relevanz von Kryptomärkten für westliche Industrienationen im Gegensatz zu den klassischen Anbau- und Produktionsstaaten wie Afghanistan, Bolivien, Kolumbien, Libanon oder Marokko, die keine bedeutende Distributionsrolle auf den anonymen Drogenmärkten im Internet spielen.

Zudem haben zahlreiche Autor_innen das Potential von Kryptomärkten zur Reduktion von schädlichen Folgen des Drogenkonsums diskutiert (vgl. Aldridge und Décary-Hétu 2014; Aldridge et al. 2017; Bancroft und Reid 2016; Barrett et al. 2016a, 2016b; Buxton und 
Bingham 2015; Caudevilla et al. 2016; Martin 2014; Tzanetakis und von Laufenberg 2016; van Buskirk et al. 2016; van Hout und Bingham 2013a, 2013b). Kryptomärkte können dazu beitragen, Schaden zu minimieren: durch die Verfügbarkeit von qualitativ hochwertigen Drogen, durch das vergleichsweise geringe Risiko einer Verunreinigung, dadurch, dass es im Vergleich zum Erwerb auf offenen Straßenmärkten kaum Gewalterfahrungen gibt (siehe unten; Hough und Natarajan 2000) und durch die Möglichkeit für Nutzer_innen, untereinander Informationen über die Qualität und Wirksamkeit der Substanzen auszutauschen. Nicht zuletzt können auf den anonymen Marktplätzen und in anonymen Foren Nutzer_innen ihr Wissen und ihre Erfahrungen an Peers weitergeben, was von Tzanetakis und von Laufenberg (2016) als peer harm reduction-Ansatz bezeichnet wird. Dessen Relevanz ist besonders bei neuen psychoaktiven Substanzen evident, zu deren kurz- und langfristigen gesundheitlichen Effekten wenig bekannt ist (vgl. van Buskirk et al. 2016).

\section{Paradoxe Kryptomärkte. Eine Analyse zentraler Veränderungs- prozesse durch die Digitalisierung von Drogenmärkten}

Die vorliegenden sozialwissenschaftlichen Erkenntnisse unterstreichen die Relevanz des Forschungsfelds der Kryptomärkte, indem sie zu einem Überblick über Angebot und Nachfrage auf diesen anonymen Märkten im Internet beitragen. Dieser Abschnitt befasst sich - unter Rückgriff darauf - mit zentralen Veränderungsprozessen, welche die Digitalisierung der Drogenmärkte mit sich bringt, sowie mit möglichen Wechselwirkungen zwischen diesen und materiellen Drogenmärkten. Unter Digitalisierung ist ein sozio-ökonomischer Wandel zu verstehen, der durch die Verbreitung digitaler Technologien sowie deren Nutzung und die dadurch bedingte Vernetzung in die Wege geleitet wurde.

\subsection{Sicherheit versus Sichtbarkeit: Reduziertes Risiko?}

Eine erste sozioökonomische Veränderung betrifft das Zusammenspiel von Risiko und Sichtbarkeit. Klassische wirtschaftswissenschaftliche Theorien erklären das traditionelle Handeln auf illegalen Drogenmärkten so, dass Händler_innen zwischen dem Risiko der Entdeckung (und Bestrafung) und einer Vergrößerung der Sichtbarkeit (mehr Transaktionen) abwägen müssen (vgl. Reuter 1983; Reuter und Kleinman 1986; Dorn et al. 2005). Eine gängige Unterscheidung auf der Einzelhandelsebene ist jene zwischen offenen, semi-öffentlichen und geschlossenen Drogenmärkten, in der das Abwägen zwischen Risiko und Sichtbarkeit zum Ausdruck kommt (vgl. Coomber 2015; Dorn et al. 1992; Hough und Natarajan 2000; May und Hough 2004; Sandberg 2012). Bei dieser Unterteilung kommt es nicht darauf an, mit welchen illegalen Drogen gehandelt wird, sondern wie sehr sich die Handelnden bei den Drogentransaktionen exponieren. Auf offenen Märkten sind die Händler_innen besonders sichtbar, sowohl für Kund_innen als auch für Strafverfolgungsbehörden. Mit der hohen Sichtbarkeit steigt das Risiko der Verhaftung und Beschlagnah- 
mung der illegalen Waren. Der Verkauf auf offenen Märkten findet im öffentlichen Raum, in Parks oder auf der Straße statt, beispielsweise bei Heroin. Semi-öffentliche Märkte stellen eine Zwischenform dar: Obwohl der Verkauf in nicht-öffentlichen Räumen wie Clubs, Cafés oder Gaststätten stattfindet, setzt er keine persönlichen Kontakte voraus. Ein Beispiel ist der Verkauf von Ecstasy in einem Club. Geschlossene Märkte sind wiederum nur jenen Konsument_innen zugänglich, welche bereits eine Vertrauensbeziehung zu den Handelnden aufgebaut haben oder durch vertrauenswürdige Bekannte vermittelt werden. Auf geschlossenen Märkten findet die Drogenübergabe an einem relativ sicheren Übergabeort im Freien oder in einer privaten Wohnung statt. Für die Handelnden ist das Risiko im Vergleich zu offenen Märkten geringer, weil sie bei der Anbahnung und Drogenübergabe weniger exponiert sind. Auf geschlossenen Drogenmärkten spielt auch die Anwendung von unterschiedlichen Formen von Gewalt eine geringere Rolle. Doch was verändert sich hierbei, wenn digitaler Technologien ins Spiel kommen?

Auf Kryptomärkten bewerben Händler_innen ihre Produkte, indem sie nicht nur Produktinformationen wie Drogenart, Menge, Preis angeben, sondern darüber hinaus auch Lieferkonditionen, Lieferdauer, Rückerstattungspolitik, Bezahlsysteme etc. (vgl. Bakken et al. 2017). Der ökonomische Erklärungsansatz für materielle Drogenmärkte greift auf Kryptomärkten nur bedingt, denn eine vergrößerte Sichtbarkeit führt hier nicht automatisch zu einem erhöhten Risiko hinsichtlich der Strafverfolgung, da Identität und Standort der Handelnden durch den Einsatz von Anonymisierungssoftware verschleiert werden. Darin liegt ein Paradoxon der Kryptomärkte und ein wichtiger Unterschied zum materiellen Drogenvertrieb: Eine Vielzahl an Handelnden kann ihre illegalen Waren auf Drogenplattformen bewerben, ohne dafür zwangsläufig strafrechtlich verfolgt zu werden. Schließlich ermöglicht es der entsprechende Einsatz von Software, den Standort und die IP-Adresse (kurz für Internet Protokoll) der Akteur_innen zu verschleiern. Zudem sind auf Online-Märkten Produkte ohne sozialen Beziehungsaufbau verfügbar, ein paar Mausklicks genügen, um die gewünschte Drogenart und Menge zu bestellen. Kryptomärkte können folglich als semi-öffentliche Drogenmärkte beschrieben werden (vgl. Tzanetakis et al. 2016).

\subsection{Hohe Verfügbarkeit und leichte Zugänglichkeit}

Die zweite Veränderung, die in diesem Beitrag diskutiert wird, betrifft Reichweite und Verfügbarkeit. Wirtschaftswissenschaftliche und soziologische Ansätze erklären die Einschränkung der geographischen Reichweite auf materiellen Drogenmärkten wie folgt: Einzelne Handelnde bzw. Netzwerke im Einzelhandelsbereich vertreiben illegale Drogen zwangsläufig mit lokaler Beschränkung (vgl. Coomber et al. 2016; Desroches 2005; Reuter 1983; Reuter und Kleinman 1986; Werse und Bernard 2016): Zum einen ist die Kontrolle von weit entfernt agierenden Mitarbeiter_innen schwer zu bewerkstelligen, womit sich das Risiko der Entdeckung durch polizeiliche Ermittlungen erhöht. Zum anderen besteht beim Transport von illegalen Waren über längere Distanzen erneut ein erhöhtes Risiko der Entdeckung durch Strafverfolgungsbehörden. Außerdem bedingt eine Ausweitung der 
Handelsradien die Zuständigkeit mehrerer Strafverfolgungsbehörden, vor allem wenn die Aktivitäten über mehrere Landesgrenzen gehen.

Analog zur Lokalität der Handelnden sind auch die Konsument_innen üblicherweise auf Händler_innen vor Ort beschränkt. Besonders bei geschlossenen Drogenmärkten haben Kund_innen eine_n Stammhändler_in oder mehrere Bezugsquellen in ihrer unmittelbaren Umgebung. Dennoch ist die Zugänglichkeit von Drogen je nach Markttypus unterschiedlich. Auch die Verfügbarkeit von psychoaktiven Substanzen variiert. Besonders auf geschlossenen Märkten kann es vorkommen, dass einzelne Drogenbestände aufgebraucht sind. In diesem Fall kann der Konsumierende entweder auf eine neue Lieferung warten oder, wenn Kontakt besteht, eine_n andere_n Händler_in aufsuchen bzw. die Ware über Freund_innen beziehen - hierbei können die Grenzen fließend sein.

Auf anonymen Drogenmärkten im Internet entfällt dagegen die geographische Beschränkung. Die technologischen Innovationen ermöglichen auf den Infrastrukturen der Plattformen weltweiten Handel mit Drogen aber auch anderen Produkten und Dienstleitungen (vgl. Tzanetakis 2018). Einschränkungen werden nur von den Händler_innen selbst vorgenommen. Der Grund für einen eingeschränkten Versand liegt in der Einschätzung des Risikos, dass internationale Postsendungen von Zollbehörden und bei Verdacht von der Polizei kontrolliert werden.

Der Zugang zu Kryptomärkten ist denkbar einfach (vgl. Tzanetakis 2017b). Neben einem Computer mit Internetanschluss ist lediglich die Aneignung des Wissens bezüglich der entsprechenden Software und der nötigen Sicherheitsvorkehrungen erforderlich. Die Digital Natives (Prensky 2001) sind die Hauptzielgruppen der anonymen Märkte im Internet, für sie ist die Aneignung dieser Fähigkeiten in der Regel einfacher als für Menschen, die erst im Erwachsenenalter den Umgang mit dem Internet und digitalen Technologien erlernt haben. Persönliche Kontakte - auf traditionellen Drogenmärkten durchaus üblich - sind hingegen nicht erforderlich. Auch beim Thema Verfügbarkeit heben sich die neuen von den traditionellen Drogenmärkten ab (vgl. Tzanetakis 2017a): Sämtliche psychoaktiven Substanzen sind auf Kryptomärkten in unterschiedlichen Mengen global verfügbar und unterliegen keiner Altersbeschränkung. Zudem sorgt der hohe Wettbewerb zwischen den Händler_innen dafür, dass Kund_innen bei Lieferproblemen bei einem bzw. einer anderen Händler_in bestellen können. Dass die Bezugsquelle unabhängig von der Örtlichkeit einfach gewechselt werden kann, ist ein Novum; wie bei sämtlichen Transaktionen im E-Commerce-Bereich genügt ein Mausklick.

\subsection{Kundenfeedback als Währung}

Eine dritte soziale Veränderung bezieht sich auf die Art und Weise, wie Vertrauen aufgebaut und Konflikte gelöst werden. Dies spielt gerade bei illegalen Märkten eine wesentliche Rolle, um das Entdeckungs- und Bestrafungsrisiko zu reduzieren. Während Transaktionen auf legalen Märkten durch den staatlich abgesicherten Schutz des Eigentumsrechts ermöglicht werden, besteht auf illegalen Märkten keine Möglichkeit für Handelspartner_ 
innen, Normen für Produktqualität oder etwa die Nichteinhaltung von Verträgen einzuklagen (vgl. Beckert und Wehinger 2013). Hinzu kommt das Risiko, für gesetzeswidriges Verhalten strafrechtlich belangt zu werden. Akteur_innen auf illegalen Märkten müssen einander also auf vielfältige Weise vertrauen, um Geschäftsaktivitäten abwickeln zu können und dabei das Risiko einer Verhaftung möglichst gering zu halten. Auf traditionellen Drogenmärkten wird Vertrauen durch interpersonelle Beziehungen meist in Form sozialer Netzwerke aufgebaut (vgl. Desroches 2005; Gambetta 2009; Paoli 2002; Reuter und Kleinman 1986; Tzanetakis et al. 2016; Werse und Bernard 2016; Zaitch 2005). Seitens des_der Käufers_in erfordert die Transaktion mit einer illegalen Ware Vertrauen und Vorsicht in Bezug auf das Verhältnis Preis/Qualität.

Auf Kryptomärkten tritt anstelle des interpersonellen ein institutionenbasiertes Vertrauen, da durch die Verwendung von Anonymisierungssoftware keine persönlichen Kontakte stattfinden. Das institutionenbasierte Vertrauen ist personenübergreifend (vgl. Zucker 1986) und basiert bei Darknet-Drogenmärkten auf dem Kundenfeedback-System und dem Bezahlsystem (vgl. Duxbury und Haynie 2018; Tzanetakis et al. 2016). Durch das Bewertungssystem können die Kund_innen ihre Einschätzung zur Produktqualität, zur vereinbarten Menge, zur Lieferdauer, zu verwendeten Verschleierungstechniken bei der Versandverpackung sowie zur Serviceorientierung des bzw. der Händlers_in zum Ausdruck bringen. Ein kurzer schriftlicher Text ist zusammen mit einem numerischen Rating für Interessierte online einsehbar und wird zusammen mit der Gesamtanzahl der Transaktionen zur Einschätzung des jeweiligen Handelnden herangezogen. Negative Bewertungen kommen äußerst selten vor, gegebenenfalls haben diese auf die Reputation etablierter Händler_innen einen geringeren Einfluss als auf Händler_innen, die z. B. weniger als ein Dutzend Bestellungen durchgeführt haben. Entscheidend ist hier die Relation der positiven zu den negativen Bewertungen.

Beim Bezahlsystem haben die Kund_innen die Wahl zwischen drei unterschiedlichen Varianten, wobei in jedem Fall mit Kryptowährungen bezahlt wird (vgl. Tzanetakis 2016a). Centralised escrow ist ein Treuhandverfahren, bei dem die virtuelle Währung erst dann für den bzw. die Händler_in freigegeben wird, wenn der bzw. die Kunde_in den Erhalt der Lieferung bestätigt hat. Das Treuhandverfahren bietet die Möglichkeit, dass der bzw. die Betreiber_in des Marktplatzes im Konfliktfall zwischen Kund_innen und Handelnden vermittelt. Während hierbei der Konsumierende vor einem möglichen Betrug abgesichert ist, kann der bzw. die Treuhänder_in den Geldbetrag unsachgemäß einbehalten. Demgegenüber wird bei Finalized early der Geldbetrag an den bzw. die Händler_in gesendet, bevor die Ware ausgeliefert wurde. Obwohl diese Variante häufig nur etablierten Händler_innen offensteht, birgt sie das Risiko, dass die Waren nach Zahlungseingang nicht versendet werden. Eine für alle Parteien gleichermaßen sichere Lösung ist Multi-signature, hier müssen zwei von drei Akteur_innen (Markt-Betreiber_in, Händler_in und Kund_in) die Zahlungsfreigabe signieren. Die Anwendung von Multi-signature ist allerdings technisch anspruchsvoller als die anderen beiden Varianten; entsprechend wird diese Bezahlvariante weniger genutzt. 
Das Kundenfeedback-System und die Bezahlsysteme auf Kryptomärkten ermöglichen also, dass Personen einander vertrauen, ohne sich persönlich zu kennen. Das tatsächlich vorhandene Vertrauen in die beiden Institutionen zeigt sich an einem Beispiel: Nach einer großangelegten Operation von internationalen Strafverfolgungsbehörden, die zur SchlieBung zahlreicher Kryptomärkte geführt hatte, gingen die Umsätze zunächst zurück. Doch bereits nach einigen Wochen wurde die Hälfte des Umsatzniveaus (vor der Operation) auf anderen Märkten wieder erreicht (vgl. Soska und Christin 2015). Die Beobachtung der Umsatzentwicklung könnte ein Indiz dafür sein, dass das Vertrauen in die Institutionen Kundenfeedback und Bezahlsystem so groß ist, dass es jenes in einzelne Marktplätze überragt. Hierzu bedarf er allerdings weiterer Forschung.

\subsection{Freier Wettbewerb?}

Eine vierte ökonomische Veränderung von illegalen Drogenmärkten betrifft die Frage des Wettbewerbs. Obwohl die Organisationsfrage je nach Staat, Drogenart, Handelsebene und Offenheit bzw. Geschlossenheit des Markts anders zu bewerten ist, wird davon ausgegangen, dass auf materiellen Drogenmärkten die Illegalität die Herausbildung großer Netzwerke verhindert (vgl. Paoli 2004; Reuter 1983; Thompson 2003). Schließlich hat die Illegalität Konsequenzen für die Organisation des Marktes und für Geschäftsbeziehungen, für das Risiko verhaftet zu werden und für die Rechtssicherheit der Akteur_innen. Die Bedingungen der Illegalität führen dazu, dass fragmentierte Marktstrukturen entstehen, wobei der Wettbewerb durch persönliche Netzwerke von lokaler Ausdehnung gekennzeichnet ist. Folglich werden materielle Drogenmärke oft als soziale Netzwerke beschrieben, die informell organisiert und relativ kurzlebig sind sowie tendenziell flache Hierarchien aufweisen (vgl. Coomber 2015; Dorn et al. 1992; Moeller und Sandberg 2015; Paoli 2004; Reuter und Kleinman 1986). Geht man von der wirtschaftswissenschaftlichen Logik aus, dass die Markteffizienz durch Wettbewerb gesteigert werden kann, dann sind illegale Märkte strukturell ineffizient (vgl. Bakken et al. 2017; Beckert und Wehinger 2013). Außerdem gibt es auf traditionellen Drogenmärkten keine staatliche Eingriffsmöglichkeit bei Preisabsprachen, auf Beschränkungen bei der Bewerbung der Waren und einen Informationsmangel in Bezug auf die Produktqualität oder auf Preise und Service.

Kryptomärkte entwickeln hingegen effizientere Marktstrukturen im Vergleich zu traditionellen Drogenmärkten (vgl. Bakken et al. 2017): Zum einen sind Transaktionen durch den Einsatz von digitaler Technologie nicht mehr an persönliche Beziehungen gebunden. Zum anderen werden psychoaktive Substanzen auf den anonymen Märkten und Foren beworben - inklusive Aktionen, um Kund_innen anzuziehen. Händler_innen informieren auf ihren Profilseiten detailliert über Produktqualität, Preise, Service und Lieferbestimmungen. Diese Angaben werden, wie oben erwähnt, von den Kund_innen bewertet und ermöglichen eine gewisse Transparenz trotz gleichzeitiger Anonymität. Demnach sind Kryptomärkte einer Selbstregulierung - basierend auf informellen Normen und Regeln - unterworfen. Allerdings fehlt ein Mechanismus gegen Preisabsprachen; außerdem kann 
nicht ausgeschlossen werden, dass unterschiedliche Profilseiten und Pseudonyme derbzw. demselben Händer_in gehören. Wettbewerb um die Gunst der Kund_innen gibt es auf Kryptomärkten auch zwischen den Händler_innen: Eine Langzeitstudie zu AlphaBay, dem ehemals größten Marktplatz hat gezeigt, dass auf diesem rund 2.200 Händler_innen etwa 12.000 unterschiedliche Drogenangebote platziert haben (vgl. Tzanetakis 2018b). Zudem gibt es auch Konkurrenz zwischen den gegenwärtig etwa ein Dutzend Kryptomärkten und Konkurrenz mit Händler_innen, die eigene Shops im Darknet betreiben.

\subsection{Der Postbote als unwissender Drogenkurier}

Die fünfte Veränderung durch die Digitalisierung von Drogenmärkten bezieht sich auf die Drogenübergabe: Wie bereits erläutert, findet der Verkauf von illegalen Drogen auf materiellen Märkten entweder öffentlich, semi-öffentlich oder geschlossen statt. Die beteiligten Akteur_innen müssen zwischen dem Grad der Sichtbarkeit bei der Drogenüber-

gabe und dem Risiko der strafrechtlichen Verfolgung abwägen, wobei sich dieses Risiko durch vertrauensvolle Handelsbeziehungen reduziert lässt

Auf Kryptomärkten, die zu den semi-öffentlichen Drogenmärkten zählen, entfällt jegliche persönliche Beziehung zwischen Händler_in und Kund_in vor, während und nach der Transaktion (vgl. Tzanetakis et al. 2016). Kund_innen haben Zugang zu einer breiten Palette an psychoaktiven Substanzen obwohl einer Transaktion keine interpersonelle Beziehung vorausgeht; das Risiko für den Handelnden wird dabei - im Gegensatz zu öffentlichen Märkten - nicht größer. Auf anonymen Drogenmarktplätzen findet zudem eine personelle, räumliche und zeitliche Trennung zwischen Verkauf und Übergabe statt. Während der Verkauf mittels virtueller Währungen online abgewickelt wird, übernimmt die Lieferung der traditionelle Post- bzw. Kurierdienst - wohlgemerkt ohne davon Kenntnis zu haben. Auf diese Art und Weise wird der Postbote zum unwissenden Drogenkurier. Einerseits entfällt damit für den bzw. die Drogenhändler_in ein Großteil des Risikos, andererseits erhöht es sich hinsichtlich der Wahl von Versandmethoden und Lieferdestinationen (vgl. Aldridge und Askew 2017; Décary-Hétu et al. 2016; Tzanetakis 2018b). Händler_innen informieren auf ihren Profilseiten darüber, welche Versandmethode (regulär, Expresszustellung, Sendungsverfolgung) sie anbieten und ob sie national, international oder gar weltweit versenden. Internationale oder weltweite Lieferungen erweitern den potentiellen Kund_innenstamm und erhöhen die Umsatzchancen; allerdings ist damit auch ein höheres Entdeckungsrisiko verbunden. Tzanetakis (2018) hat dokumentiert, dass etwa die Hälfte der Händler_innen ein höheres Risiko einzugehen bereit sind und weltweit Drogen versenden, während die andere Hälfte der Händler_innen den Versand innerhalb ihrer Region bevorzugt. Insofern gibt es bei Drogenübergabe eine Parallele zwischen Kryptomärkten und materiellen Drogenmärkten, da auf beiden die erhöhte Sichtbarkeit (und damit die Chance auf höhere Umsätze) mit einem erhöhten Entdeckungsrisiko einhergeht. 


\section{$4 \quad$ Perspektiven und Regulierungsansätze}

In diesem Beitrag werden sozioökonomische Folgen und Grenzen der zunehmenden Verbreitung digitaler Technologien für illegale Drogenmärkte dargelegt. Basierend auf sozialwissenschaftlichen Forschungserkenntnissen konnten grundlegende Veränderungen durch die Digitalisierung von Märkten und der damit einhergehenden Herausbildung von Kryptomärkten herausgearbeitet werden. Durch die Verwendung von Anonymisierungssoftware können Handelnde auf anonymen Plattformen im Internet ihre Sichtbarkeit erhöhen, ohne dass gleichzeitig das Risiko der Strafverfolgung steigt. Ebenso sind anonyme Drogenmärkte ohne persönlichen Beziehungsaufbau, dafür nach Aneignung des technischen Wissens, global verfügbar und haben keine zeitliche Begrenzung und keine Altersbeschränkung. Hinzu kommt, dass auf Kryptomärkten anstelle des interpersonellen ein institutionenbasiertes Vertrauen hergestellt wird, etwa durch Feedback- oder Bezahlsysteme. Durch Wettbewerb zwischen Handelnden und Marktplätzen wird die Transparenz in Bezug auf die Qualität der angebotenen Drogen stellenweise erhöht. Außerdem wird auf Kryptomärkten die Drogenübergabe von traditionellen Post- bzw. Kurierdiensten übernommen, ohne dass diese davon Kenntnis haben.

Doch welche wirtschaftliche Relevanz hat das neue Phänomen des Drogenhandels über das Darknet? Der Anteil am gesamten Einzelhandelsmarkt für illegale Drogen ist klein, wobei für die Zukunft von einer steigenden Relevanz des Internets und digitaler Technologien für den Drogenvertrieb auszugehen ist. Bereits heute zeigt sich, dass Kryptomärkte im Gegensatz zu Straßenmärkten einerseits den Vertreib qualitativ hochwertiger Inhaltsstoffe ermöglichen und andererseits die höhere Sichtbarkeit von Handelnden nicht zwangsläufig mit einem erhöhten Verhaftungsrisiko korreliert.

Im Einklang mit der Auflösung von Raum-Zeit-Handelsbeziehungen in modernen kapitalistischen Ökonomien (Giddens 1990) steht einer Expansion von Marktbeziehungen auch auf Kryptomärkten, nichts mehr im Wege. Während institutionenbasiertes Vertrauen auf materiellen, illegalen Märkten nur beschränkt möglich ist, erlauben die Fortschritte in der Kommunikationstechnologie die Herausbildung ebendieses Vertrauenstyps auf anonymen Plattformen zum Vertrieb illegaler Waren. Trotz der sich weltweit erstreckenden und in Konventionen gegossenen Drogenprohibition haben sich durch die Digitalisierung Kryptomärkte entwickelt, auf denen Praktiken einer institutionellen Selbstregulierung etabliert sind. Diese erlauben eine Ausweitung von Marktbeziehungen auf der Basis von effizienteren Marktstrukturen, obwohl Strafverfolgungsbehörden das Verbot umsetzen. Damit werden Kryptomärkte strukturell gesehen zu dynamischen Wachstumsmärkten.

Selbstregulierung bedeutet nach wirtschaftstheoretischem Verständnis idealtypischer Weise, dass Angebot auf Nachfrage trifft und Waren auf der Basis von Preisen getauscht werden, die den Nutzen aller an der Tauschbeziehung beteiligten Akteur_innen maximieren. Doch in der Praxis kommt es immer wieder zu Marktkonzentrationen. Zudem gibt es Überschneidungen von materiellen und virtuellen Drogenmärkten: Rund ein Viertel der Transaktionen sind aufgrund ihrer Größe nicht für den Eigenkonsum bestimmt und werden teilweise profitorientiert weiterverkauft. Kauft jemand für den Eigenkonsum auf 
Kryptomärkten, kann die Person im Vergleich zum materiellen Einzelhandel qualitativ hochwertigere Substanzen, allerdings zu einem höheren Preis erwerben. Kauft allerdings ein_e Einzelhändler_in online mit der Intention, die Drogen weiterzuverkaufen, kann die Qualität ein kaufentscheidendes Kriterium sein - muss es aber nicht. Wahrscheinlicher ist hingegen, dass der Preis die Kaufentscheidung bestimmt. Die sich daraus ergebenden Gefahren für Konsumierende benötigen ebenso eine entsprechende Regulierung. Eine Besonderheit der Kryptomärkte liegt darin, dass sie bestehende und global geltende Anti-Drogenkonventionen systematisch umgehen und gleichzeitig eine Dimension von Tauschbeziehungen ermöglichen, die davor nicht denkbar war: der global vernetzte Handel mit illegalisierten Rauschmitteln.

Vor diesem Hintergrund wird ein Nachdenken über eine Regulierung und Entkriminalisierung von Drogen wichtiger denn je, denn diese entziehen dem neuen Phänomen den Nährboden. Dabei müssen Antworten auf mindestens drei Fragen gefunden werden: Wie kann es staatlichen Behörden gelingen, die Herausbildung von kartellartigen Handelsstrukturen zu verhindern und das Marktgeschehen on- wie offline zu regulieren? Mit welchen Maßnahmen in den Bereichen der Prävention, Beratung und Betreuung, können Drogenkonsumierende entkriminalisiert werden? Dies gilt für besonders problematisches Konsumverhalten, die Umsetzung einer entsprechenden Altersbeschränkung für virtuelle wie materielle Transaktionen sowie niedrigschwellige Drogenarbeit etwa durch die Kombination onlinebasierter und konventioneller Drogenberatungs- sowie analysegestützter Interventionen (z.B. Drug Checking-Angebote). Wie gestaltet sich eine angemessene (und vor allem auch angemessen finanzierte) sozialwissenschaftliche Begleitforschung, die in der Lage ist, die sozialen und kulturellen Dimensionen der eingesetzten Maßnahmen sowie unbeabsichtigte Folgen zu erheben und deren Wirksamkeit zu evaluieren?

\section{Literatur}

Aldridge, J., D. Décary-Hétu. 2014. Not an 'Ebay for Drugs': The Cryptomarket 'Silk Road' as a Paradigm Shifting Criminal Innovation. doi: http://dx.doi.org/10.2139/ssrn.2436643.

Aldridge, J., D. Décary-Hétu. 2016. Hidden wholesale: The drug diffusing capacity of online drug cryptomarkets. International Journal of Drug Policy 35: 7-15.

Aldridge, J., A. Stevens, M. J. Barratt. 2017. Will growth in cryptomarket drug buying increase the harms of illicit drugs? Addiction. doi: 10.1111/add.13899.

Bakken, S., K. Moeller, S. Sandberg. 2017. Coordination problems in cryptomarkets: Changes in cooperation, competition and valuation. European Journal of Criminology. doi: 10.1177/1477370817749177.

Bancroft, A., P. S. Reid. 2016. Concepts of illicit drug quality among darknet market users: Purity, embodied experience, craft and chemical knowledge. International Journal of Drug Policy 35: 42-49.

Barratt, M. J., J. A. Ferris, A. R. Winstock. 2014. Use of Silk Road, the online drug marketplace, in the UK, Australia and the USA. Addiction 109: 774-783. 
Barratt, M. J., A. Maddox, S. Lenton, M. Allen. 2016a. What if you live on top of a bakery and you like cakes?-Exploring the drug use and harm trajectories before, during and after the emergence of Silk Road. International Journal of Drug Policy 35: 50-57.

Barratt, M. J., J. A. Ferris, A. R. Winstock. 2016b. Safer scoring? Cryptomarkets, social supply and drug market violence. International Journal of Drug Policy 35: 24-31.

Beckert, J., F. Wehinger. 2013. In the shadow: Illegal markets and economic sociology. Socio-Economic Review 11: 5-30.

Buxton, J., T. Bingham. 2015. The rise and challenge of dark net drug markets. Policy Brief 7. Global Drug Policy Observatory. Zugegriffen: 15. Januar 2018.

Caudevilla, F., M. Ventura, I. Fornís, M. J. Barratt, C. G. lladanosa, P. Quintana, A. Munoz, N. Calzada. 2016. Results of an international drug testing service for cryptomarket users. International Journal of Drug Policy 35: 38-41.

Christin, N. 2013. Traveling the Silk Road: A Measurement Analysis of a Large Anonymous Online Marketplace. In Proceedings of the 22nd international conference on World Wide Web (WWW '13) 213-224. Rio de Janeiro.

Coomber, R. 2015. A Tale of Two Cities Understanding Differences in Levels of Heroin/Crack Market-Related Violence-A Two City Comparison. Criminal Justice Review 40: 7-31.

Coomber, R., L. Moyle, N. South. 2016. Reflections on three decades of research on social supply in the UK. In Friendly Business. International views on social supply, self-supply and small-scale drug dealing, hrsg. v. B. Werse, C. Bernard, 13-28. Wiesbaden.

Darknet Stats. https://dnstats.net/ Zugegriffen: 26. Januar 2018.

Demant, J., R. Munksgaard, E. Houborg. 2016. Personal use, social supply or redistribution? Cryptomarket demand on Silk Road 2 and Agora. Trends in Organized Crime: 1-20.

Desroches, F. 2005. The crime that pays: Drug trafficking and organized crime in Canada. Toronto.

Dorn, N., K. Murji, N. South. 1992. Traffickers: Drug Markets and Law Enforcement. London.

Dorn, N., M. Levy, L. King. 2005. Literature Review on Upper Level Drug Trafficking. Home Office Online Report 22/5. London.

Dorn, N., K. Murji, N. South. 1992. Traffickers: Drug Markets and Law Enforcement. London.

Duxbury, S. W., D. L. Haynie. 2018. Building them up, breaking them down. Topology, vendor selection patterns, and a digital drug market's robustness to disruption. Social Networks 52: 238-250.

European Monitoring Centre for Drugs and Drug Addiction and Europol (EMCDDA). 2016. EU Drug Markets Report. In-Depth Analysis. EMCDDA-Europol Joint publications. Luxembourg.

Gambetta, D. 2009. Codes of the Underworld: How Criminals Communicate. Princeton, Oxford.

Giddens, A. 1990. The Consequences of Modernity. Stanford (CA).

Hough, M., M. Natarajan. 2000. Introduction: Illegal Drug Markets, Research and Policy. In Illegal Drug Markets: From Research to Prevention Policy, Crime Prevention Studies, hrsg. v. M. Natarajan, M. Hough, 1-17. Monsey (NY).

Kruithof, K., J. Aldridge, D. Décary-Hétu, M. Sim, E. Dujso, S. Hoorens. 2016. Internet-facilitated drugs trade. An analysis of the size, scope and the role of the Netherlands. Santa Monica (CA), Cambridge.

Martin, J. 2014. Drugs on the Dark Net. How Cryptomarkets are Transforming the Global Trade in Illicit Drugs. Basingstoke.

May, T., M. Hough. 2004. Drug markets and distribution systems. Addiction Research \& Theory 12: $549-563$.

Martin, J. 2014. Drugs on the Dark Net. How Cryptomarkets are Transforming the Global Trade in Illicit Drugs. Basingstoke.

Moeller, K., S. Sandberg. 2015. Credit and trust management of network ties in illicit drug distribution. Journal of Research in Crime and Delinquency 52: 691-716. 
Ormsby, E. 2016. Silk Road: insights from interviews with users and vendors. In EU Drug Markets Report. In-Depth Analysis, hrsg. v. EMCDDA, 61-68. Luxembourg.

Paoli, L. 2002. Flexible Hierarchies and Dynamic Disorder: the Drug Distribution System in Frankfurt and Milan. Drugs: Education, Prevention and Policy 9: 143-151.

Paoli, L. 2004. „Die unsichtbare Hand des Marktes“. Illegaler Drogenhandel in Deutschland, Italien und Russland. In Soziologie der Kriminalität, hrsg. v. D. Oberwittler, S. Karstedt, 356-383. Wiesbaden.

Prensky M. 2001. Digital natives, digital immigrants Part 1, On the Horizon 9: 1-6. doi: 10.1108/10748120110424816.

Reuter, P. 1983. Disorganized crime: the economics of the visible hand. Cambridge (MA).

Reuter, P., M. A. Kleiman. 1986. Risks and prices: an economic analysis of drug enforcement. Crime and Justice 7: 289-340.

Sandberg, S. 2012. The Importance of Culture for Cannabis Markets Towards an Economic Sociology of Illegal Drug Markets. British Journal of Criminology 52: 1133-1151.

Soska, K., N. Christin. 2015. Measuring the Longitudinal Evolution of the Online Anonymous Marketplace Ecosystem. In Proceedings of the 24th USENIX Security Symposium, August 12-14, 2015, 33-48. Washington, DC.

Taylor, M., G. R. Potter. 2013. From "Social Supply" to "Real Dealing”: Drift, Friendship, and Trust in Drug Dealing Careers. Journal of Drug Issues 43: 392-406.

Thompson, G. 2003. Between Hierarchies and Markets: The Logic and Limits of Network Forms of Organization. Oxford.

Tzanetakis, M. 2016a. Online drug distribution: alternatives to physical violence in conflict resolution. In Between street and screen. Traditions and innovations in the drugs field, hrsg. v. M. Wouters, J. Fountain, 41-56. Lengerich.

Tzanetakis, M. 2016b. Von der visuellen Symbolik zum Vertrauen schaffenden System der virtuellen Währung Bitcoin. In Der schöne Schein. Symbolik und Ästhetik von Banknoten, hrsg. v. S. Hartmann, C. Thiel, 273-299. Regenstauf.

Tzanetakis, M. 2017a. Drogenhandel im Darknet. Gesellschaftliche Auswirkungen von Kryptomärkten. Aus Politik und Zeitgeschichte 67: 41-46.

Tzanetakis, M. 2017b. Zur globalen Ökonomie von digitalen Drogenmärkten. Rausch - Wiener Zeitschrift für Suchttherapie 6: 190-199.

Tzanetakis, M. 2018a. The darknet's anonymity dilemma. In Encore 2017. The Annual Magazine on Internet and Society Research, hrsg. v. Alexander von Humboldt Institute for Internet and Society, 118-125. Berlin.

Tzanetakis, M. 2018b. Comparing cryptomarkets for drugs. A characterisation of sellers and buyers over time, International Journal of Drug Policy, Special Issue 'Comparing Drug Policies'. doi: https://doi.org/10.1016/j.drugpo.2018.01.022.

Tzanetakis, M., G. Kamphausen, B. Werse , R. v. Laufenberg. 2016. The transparency paradox. Building trust, resolving disputes and optimising logistics on conventional and online drugs markets. International Journal of Drug Policy 35: 58-68.

Tzanetakis, M., H. Stöver. (Hrsg.). 2018. Drogen, Darknet und Organisierte Kriminalität. Baden-Baden (im Erscheinen).

Tzanetakis, M., R. v. Laufenberg. 2016. Harm Reduction durch anonyme Drogenmärkte und Diskussionsforen im Internet? In 3. Alternativer Drogen- und Suchtbericht, hrsg. v. akzept e.V. Bundesverband für akzeptierende Drogenarbeit und humane Drogenpolitik, 189-194. Lengerich.

Van Buskirk, J., S. Naicker, A. Roxburgh, R. Bruno, L. Burns 2016. Who sells what? Country specific differences in substance availability on the Agora dark net marketplace. International Journal of Drug Policy 35: 16-23. 
Van Hout, M. C., T. Bingham. 2013a. 'Silk Road', the virtual drug marketplace: A single case study of user experiences. International Journal of Drug Policy 24: 385-391.

Van Hout, M. C., T. Bingham. 2013b. 'Surfing the Silk Road': A study of users' experiences. International Journal of Drug Policy 24: 524-529.

Werse, B., C. Bernard. (Hrsg.). 2016a. Friendly Business - International Views on Social Supply, Self-Supply, and small-scale Drug Dealing. Wiesbaden.

Zaitch, D. 2005. The ambiguity of violence, secrecy, and trust among Colombian drug entrepreneurs. Journal of Drug Issues 35: 201-228.

Zucker, L. G. 1986. Production of Trust: Institutional Sources of Economic Structure, 1840-1920. Research in Organizational Behavior 8: 53-111.

Open Access Dieses Kapitel wird unter der Creative Commons Namensnennung 4.0 International Lizenz (http://creativecommons.org/licenses/by/4.0/deed.de) veröffentlicht, welche die Nutzung, Vervielfältigung, Bearbeitung, Verbreitung und Wiedergabe in jeglichem Medium und Format erlaubt, sofern Sie den/die ursprünglichen Autor(en) und die Quelle ordnungsgemäß nennen, einen Link zur Creative Commons Lizenz beifügen und angeben, ob Änderungen vorgenommen wurden.

Die in diesem Kapitel enthaltenen Bilder und sonstiges Drittmaterial unterliegen ebenfalls der genannten Creative Commons Lizenz, sofern sich aus der Abbildungslegende nichts anderes ergibt. Sofern das betreffende Material nicht unter der genannten Creative Commons Lizenz steht und die betreffende Handlung nicht nach gesetzlichen Vorschriften erlaubt ist, ist für die oben aufgeführten Weiterverwendungen des Materials die Einwilligung des jeweiligen Rechteinhabers einzuholen

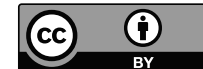

